\title{
Obsessive-Compulsive Spectrum Phenomena and the DSM-V Developmental Process
}

\author{
By Eric Hollander, MD
}

Several of this month's articles and interviews touch on themes that relate to spectrum phenomena as well as the Diagnostic and Statistical Manual of Mental Disorders developmental process.

First, Darrel A. Regier, MD, MPH, director of the Division of Research at American Psychiatric Association, discusses, in an interview with CNS Spectrums, the developmental process for $D S M-V$. He emphasizes the use of dimensional measures to determine both thresholds for disorders, and to assess response to treatments. He also highlights a focus on spectra of disorders that cut across traditional diagnostic boundaries as one way to deal with issues of comorbidity. Finally, he discusses new approaches to the five $D S M$ axes, and the need to link together the DSM and International Classification of Diseases processes. Three other articles in this issue also clearly relate to these obsessive-compulsive spectra issues.

For example, Leonardo F. Fontenelle, MD, PhD, describes how, although much attention has been paid to patients who lack insight into their obsessional beliefs, less importance has been given to individuals with obsessive-compulsive disorder (OCD) who display perceptual disturbances typically found in psychotic disorders, including schizophrenia, schizoaffective disorders, or mood disorders with psychotic features. The authors call attention to a phenomenon that has been neglected in the psychiatric literature (ie, the occurrence of hallucinations and related phenomena in patients with $O C D$ ). They describe five patients with OCD with hallucinations in several different sensory modalities, including the auditory, the visual, the tactile, the olfactory, and the cenesthetic modalities, and suggest that further psychopathological research should clarify the clinical significance of hallucinations among patients with OCD.

Andrea Allen, $\mathrm{PhD}$, and colleagues describe the first known study of the serotonin norepinephrine reuptake inhibitor venlafaxine in body dysmorphic disorder (BDD). BDD, a preoccupation with imagined ugliness, is a disabling condition that is considered in the $D S M-V$ process as a putative obsessive-compulsive spectrum disorder. It seems to respond preferentially to selective serotonin reuptake inhibitors. The authors studied 17 BDD patients who participated in a 12-16-week open-label trial of venlafaxine, using the Yale-Brown Obsessive-Compulsive Scale Modified for BDD and Clinical Global Impressions-Improvement scale as outcome measures. Venlafaxine was found to be effective in lessening specific obsessive and compulsive symptoms of BDD and global severity of BDD. Of course, controlled research on venlafaxine in $\mathrm{BDD}$ is recommended.

Chih-Hung Ko, MD, and colleagues studied internet addiction, which has been proposed in the $D S M$ process as a potentially new impulse control disorder. This condition has been conceptualized as an externalizing disorder, a behavioral addiction, or an obsessive-compulsive spectrum disorder. The study examined the association between Internet addiction and comorbid conditions, such as depressive disorder, social phobia, and adult attention-deficit/hyperactivity disorder (ADHD), and examined gender differences in psychiatric comorbidity in a sample of Taiwanese college students. Both adult ADHD and depressive disorders were associated with Internet addiction. However, depressive disorders were

Dr. Hollander is the editor of this journal, Esther and Joseph Klingenstein Professor and Chairman of Psychiatry at the Mount Sinai School of Medicine, and director of the Seaver and New York Autism Center of Excellence in New York City. 
associated with Internet addiction only in males. Thus, college students with Internet addiction, a growing problem, should be evaluated and treated for adult ADHD and depressive disorders. It is important to keep in mind that comorbidity has an important influence on presentation, course of illness, and treatment response in various spectra disorders.

Diphenhydramine, an over-the-counter antihistamine, is used by millions of people in the United States for its sedating properties as a hypnotic. Occasionally patients respond with paradoxical excitation, but there are no studies on the prevalence of paradoxical excitation in healthy subjects. One percent to $2 \%$ of the US population may have at least three active copies of the cytochrome P450 (CYP) 2D6 gene, are ultrarapid metabolizers (UMs), and present high metabolic capacity for CYP2D6 substrates. Jose de Leon, MD, and D. Michele Nikoloff, PhD, describe three
CYP2D6 UMs who reported paradoxical excitation on diphenhydramine. They suggest that in CYP2D6 UMs, diphenhydramine may be converted to a compound that causes excitation due to the abnormally high CYP2D6 activity. More formal studies of diphenhydramine responses in CYP2D6 UMs are required to test this.

Finally, in a communique, Kim J. Masters, MD, describes clinical observations in an adolescent treatment center that lithium seems to reduce both suicidal thoughts, and self-harm behaviors, such as cutting. Jennifer M. Rosenberg, MD, MPH, and Carl Salzman, MD, who had previously reviewed new uses for lithium in anxiety, substance use, and psychotic disorders, respond that lithium may also reduce suicidal behaviors in bipolar patients.

May you stay warm and informed during the winter months. CNS

\section{Now Available Online at www.cnsspectrums.com CME-ACCREDITED SUPPLEMENT $13 \mathrm{cme}$ An expert review of clinical challenges in psychiatry \\ Case Studies in Bipolar Depression: Best Practices for the Hospitalized Patient

\author{
by Paul E. Keck, Jr, MD, Mark A. Frye, MD, and Michael E. Thase, MD
} \\ To request a published supplement, pleasee-mail ks@mblcommunications.com \\ Supported by an educational grant from AstraZeneca.}

